\title{
A Conservative Approach to the Management of a Dental Trauma for Immediate Natural Esthetics
}

\author{
Pallav Mahesh Patni, ${ }^{1,}$ Pradeep Jain, ${ }^{2}$ and Mona Jain Patni ${ }^{3}$ \\ ${ }^{1}$ Department of Conservative Dentistry and Endodontics, Sri Aurobindo College of Dentistry, Sri Aurobindo Institute of Medical Sciences and Hospital (SAIMS), Devi Ahilya \\ University, Indore, Madhya-Pradesh, India \\ ${ }^{2}$ Department of Conservative Dentistry and Endodontics, Sri Aurobindo Institute of Medical Sciences and Hospital, Indore, Madhya-Pradesh, India \\ ${ }^{3}$ Cosmetic dentistry, Dr Mahesh Chandra Patni memorial dental clinic, Indore, Madhya-Pradesh, India \\ "Corresponding author: Pallav Mahesh Patni, Department of Conservative Dentistry and Endodontics, Sri Aurobindo College of Dentistry, Sri Aurobindo Institute of Medical \\ Sciences and Hospital (SAIMS), Devi Ahilya University, Indore, Madhya-Pradesh, India. Tel: +91-9993060025, E-mail: pallavpatni@yahoo.com
}

Received 2015 April 14; Revised 2015 June 09; Accepted 2015 June 24.

\begin{abstract}
Introduction: The fracture of front teeth is one of the routine presentations of traumatic injuries. The treatment of a fractured tooth involving the pulp includes root canal therapy and post placement followed by core build-up or by the extraction of the fractured tooth if it is not restorable.

Case Presentation: We report a case of an adult male who had traumatized both his maxillary central incisors following a blow experienced during domestic violence. He had lost a fractured fragment of the right central incisor, while the left incisor had complicated fractures with fragments retained attached to the soft tissue. Following radiovisiography (RVG), both incisors were conservatively treated in a single visit by reattachment and post and core techniques.

Conclusions: The treatment reported for reattachment of the tooth fractures and post and core techniques are reasonably easy while providing immediate and lasting results in patients' regaining of social confidence and functionality.
\end{abstract}

Keywords: Composite Resins, Esthetics, Tooth Fractures, Wounds and Injuries

\section{Introduction}

A traumatic injury with the fracture of the front teeth is a dreadful experience for the patient. This condition requires immediate consideration for both esthetics and functionality. Dental trauma primarily leads to fracture of the front teeth, specifically the upper incisors, because of their location in the mouth. In road accidents, domestic violence, and sports injuries, multiple and/or complicated tooth fractures may be observed (1).

Many procedures have been used to preserve the fractured teeth, initially including crowns, orthodontic bands, pins, and a full crown (2). Tennery (1988) (3) was the first to report the reattachment of a fractured tooth. Similarly, several others have described success with reattachment cases $(4,5)$. The use of composites as adhesives to the tooth made reattachment, with conservative management, promising for fractured teeth $(6,7)$. Reattachment procedures can be used with a successful outcome on both uncomplicated and complicated fractures $(8,9)$.

\section{Case Presentation}

A 42-year-old male reported to a dental clinic immediately following a trauma due to domestic violence. Care- ful examination revealed no other injury except the fracture of the upper front teeth. The crown portion of the maxillary left central incisor had been fractured and had not been retained by the patient. Clinical examination revealed the presence of a horizontal fracture at the cervical level. The right central incisor had complicated vertical and oblique fractures, and the fragments were retained, attached to the soft tissues (Figure 1A). Exposed pulp was examined clinically and was confirmed radiographically (Figure 1B). Hence, root canal treatment was planned. The presence of normal occlusion was confirmed. The fractured fragment of the right central incisor was removed (Figure 1C and D). Normal root condition was assessed with preoperative radiographs.

Following radiographic assessment, the following treatment plan was presented to the patient:

1. Endodontic treatment and reattachment of the fractured tooth fragment of the left central incisor;

2. A post and core treatment followed by full veneer restorations of the right central incisor.

The treatment plan was decided as follows: the completion of endodontic treatment of both central incisors, the reattachment of the fractured fragment of the left central

Copyright (c) 2016, Kashan University of Medical Sciences.. This is an open-access article distributed under the terms of the Creative Commons Attribution-NonCommercial 4.0 International License (http://creativecommons.org/licenses/by-nc/4.0/) which permits copy and redistribute the material just in noncommercial usages, provided the original work is properly cited. 

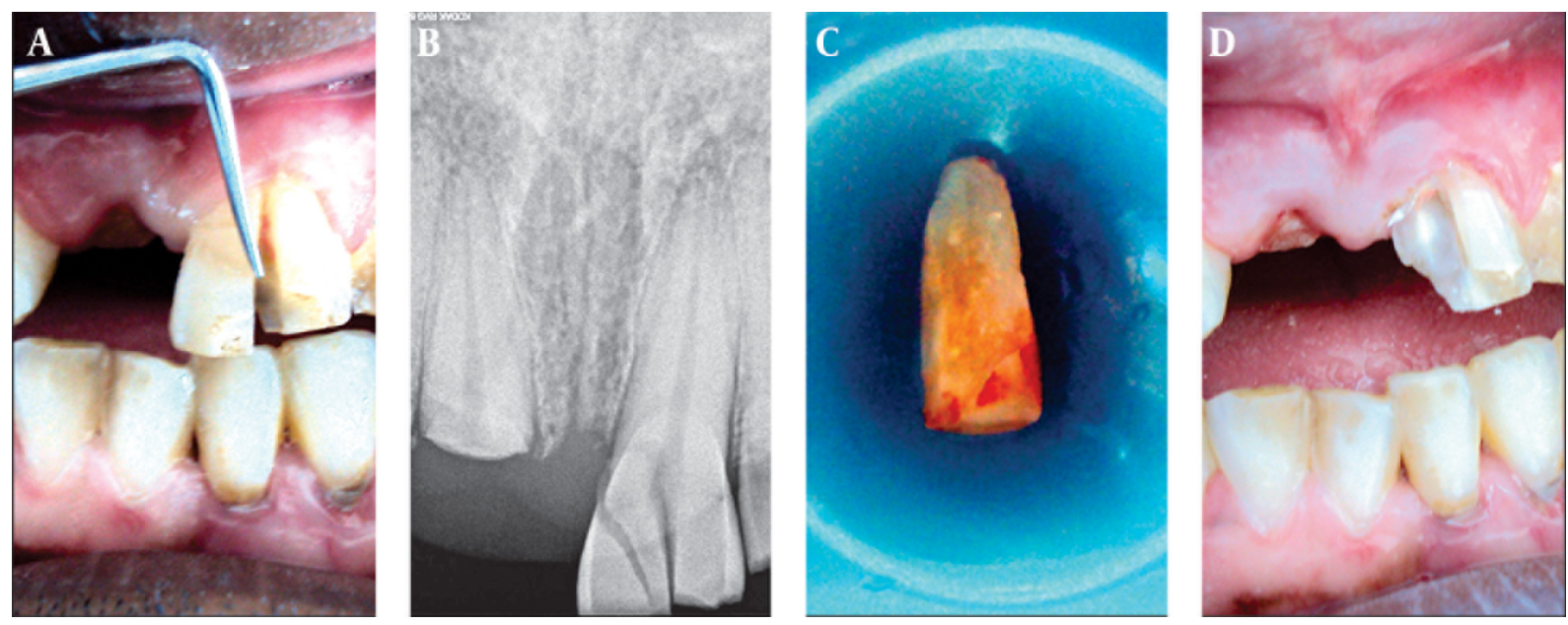

A, Preoperative photograph of both fractured maxillary central incisors; B, Preoperative radiograph revealing fracture of both maxillary central incisors; C, Removed fractured fragment of left maxillary incisor; D, Left maxillary incisor after removal of fractured fragment.

incisor, and the preparation of a post space followed by the cementation of the post and the core build-up of the right central incisor. The fractured fragment from the left central incisor was placed in 5\% sodium hypochlorite for two minutes to clear the pulp tissue remnants and was stored in normal saline before the commencement of the treatment.

The endodontic treatment was performed on both incisors. Following isolation using a rubber dam, an endodontic access opening was made under the magnification and illumination of a dental operating microscope (8x, Global Surgical Corporation, St. Louis MO, USA); using an endo-access bur (Dentsply Maillefer, USA), conventional access was prepared. Then patency was checked using 10 K-files (Kerr USA), and the working length was determined by using an electronic apex locator Root ZX mini (J. Morita MFG. Corporation, Kyoto, Japan) and a radiograph with an endodontic file inserted in the canal.. The root canals were cleaned and shaped by a Rotary Ni-Ti Protaper system (Dentsply Maillefer, USA) along with Glyde (Dentsply Maillefer, USA), using a crown down technique. The root canals were copiously irrigated with 5.2\% sodium hypochlorite and 17\% EDTA solution during the preparation. Following this, a final irrigation was done with a $2 \%$ chlorhexidine solution (V-concept, Vishal Dentocare, India). The root canals were dried using paper points. Before obturation, master points were seated to test their suitability to canals, and a radiograph was taken.

Both maxillary central incisors were obturated with the selected master gutta-percha cone (Protaper, Dentsply Maillefer) and sealer (AH-Plus, Dentsply, Maillefer, USA).
The coronal gutta-percha cones were seared off using a heated instrument, and vertical compaction was done using heated pluggers at the individual canal orifices.

Reattachment of the fractured tooth fragment of the left central incisor was carried out using a dual-cure composite resin cement (Multi-Link Automix, Ivoclar Vivadent AG, Schaan/Liechtenstein), and the crown of the restored tooth was created using Luxacore (DMG Dental Avenue India) (Figure $2 \mathrm{~A}$ and $\mathrm{B}$ ).

A post space was prepared in the radicular portion of the right central incisor using the glass fiber FRC Postec Plus system (FRC Postec Plus Ivoclar Vivadent AG, Schaan/Liechtenstein). An appropriately sized post was cemented using dual-cure composite resin cement (MultiLink Automix, Ivoclar Vivadent AG, Schaan/Liechtenstein) (Figure 2C) .The core build-up was subsequently done using Luxacore (DMG Dental Avenue India), a dual cured core build-up material.

The post and core were checked for the absence of mobility, and a radiograph was taken (Figure 2D). The restoration after final finishing was checked for occlusal harmony (Figure 2E). The complete procedure was carried out in a single visit, as the patient demanded immediate esthetics due to some social commitment later on the same day.

At a follow-up visit after five years and four months, both the incisors were firm, and the treatment outcome was found to be successful (Figure 2F). 

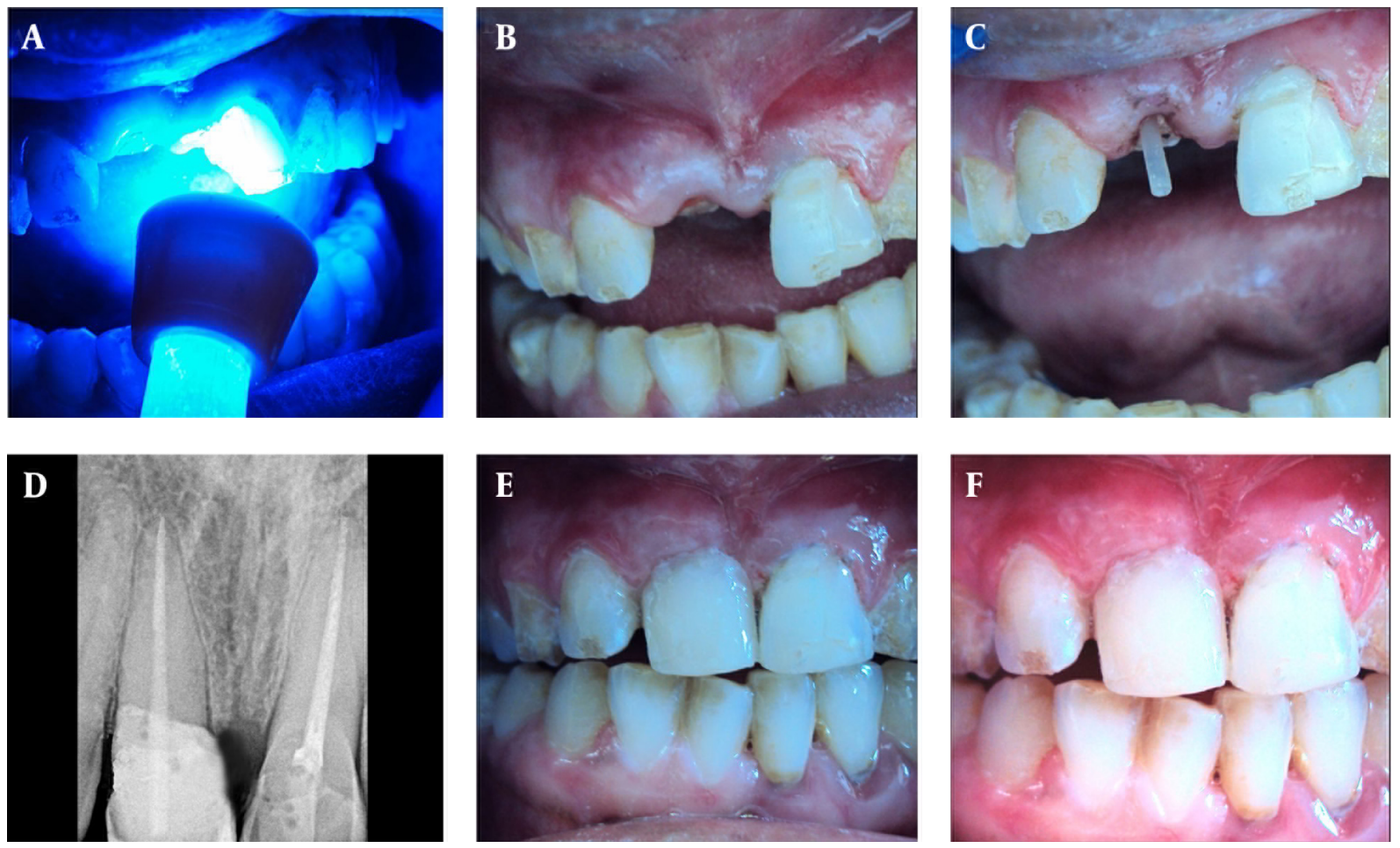

A and B, Reattachment of fractured fragment of left central incisor; C, Post space preparation and post placement of right central incisor; D, Postoperative radiograph; E, Postoperative photograph; F, Clinical examination done at follow-up after 5 years and 4 months revealed a stable reattachment of the fragment and good esthetics.

\section{Discussion}

The study of several case reports shows that more than $80 \%$ of fractured anterior teeth separate obliquely from the buccal to the palatal side, and the fracture progresses toward the apical area. This fact has been confirmed in an experimental study (10). A good reattachment of the fractured incisor is often an intimidating clinical condition. But reattachment has many benefits:

1. Original esthetics, as the actual fractured tooth fragment is reattached.

2. Tooth attrition is similar to that of other natural teeth.

3. Reattachment is less time-consuming than other prosthodontic or implant options.

4. A positive response from the patient for reattaching original tooth fragment.

In the case presented, the pulp was involved with both the incisors, and hence, root canal treatment was performed. Post placement and composite resin bonding were used to achieve adequate retention and to resist various occlusal forces. In the present case, the fracture was relatively simple and supragingival, and hence, no treatment such as extrusion was required. van Dijken et al. (11) stated that composite resin has a favorable subgingival response.

The FRC Postec Plus system (FRC Postec Plus Ivoclar Vivadent AG, Schaan/Liechtenstein) consists of glass fibers embedded in a composite matrix. This design produces a post with an elastic behavior (modulus of elasticity) similar to that of dentin, unlike metal or ceramic posts. Hence, in the presented case, an FRC Postec Plus post was used for the retention of the core build-up (7).

The treatments performed in the present case report were practical and simple while reinstating purpose, looks, and confidence with very conservative management. However, the clinician has to maintain isolation protocols and should adhere to the manufacturer's instructions to attain optimum results in bonding. Several case reports and studies have proved that reattachment of a fractured tooth fragment is a viable and preferred treatment where indicated, and long-term success can be achieved (12-16). 
The significant innovation of adhesive methods and resin composites has made reattachment of tooth fragments a procedure that is no longer a provisional restoration, but rather, a restorative treatment offering a promising prognosis. However, this practice can be used only when the undamaged tooth fragment is obtainable (13).

Although immediate and desirable esthetics were obtained in a single visit owing to the patient's time constraints, the tooth with the reattached fragment served optimum esthetics and stability on the follow-up visit. The patient did not opt for a prosthesis for the tooth with post and core during the follow-up visit.

In the case presented, the factors contributing to success may be the optimum fit and adaptation of the fractured fragment, root integrity, and a successful bonding technique. With the advanced bonding adhesives currently available, these, in combination with suitable procedures and skill, can achieve superior visual outcomes.

In the presented case, over a long-term follow-up, restoration of the traumatized teeth through the use of an adhesive material in conjunction with a prefabricated post appeared to be a successful technique. A quick esthetic outcome was attained, and the lack of intermediary laboratory processes made this treatment less expensive and less time-consuming.

\section{Acknowledgments}

The authors acknowledge Dr. Manjushree Bhandari, Chairperson, SAIMS, and Dr. Mahak Bhandari, Director, Mohak Hi-tech Hospitals, for their kind guidance and support.

\section{Footnote}

Authors' Contribution: Pallav Mahesh Patni: Developed the original idea, developed the protocol, and abstracted and analyzed the data. Pradeep Jain: Developed the protocol, abstracted the data, and prepared the manuscript. Mona Jain Patni: Abstracted and analyzed the data, prepared the manuscript, and contributed to the development of the protocol.

\section{References}

1. Olsburgh S, Jacoby T, Krejci I. Crown fractures in the permanent dentition: pulpal and restorative considerations. Dent Traumatol. 2002;18(3):103-15. [PubMed: 12110103]

2. Simonsen R, Thompson VP, Barrark G. Etched cast restorations:clinical and laboratory technique. Chicago: Quintessence; 1985. pp. $150-1$.

3. Tennery TN. The fractured tooth reunited using the acid-etch bonding technique. Tex Dent J. 1978;96(8):16-7. [PubMed: 295156].

4. Davari A, Sadeghi M. Influence of different bonding agents and composite resins on fracture resistance of reattached incisal tooth fragment. J Dent (Shiraz). 2014;15(1):6-14. [PubMed: 24738084].

5. Kim B, Lee Y, Song MJ, Shin SJ, Park JW. Considerations during crown reattachment procedure over the pulpal exposure: case report. Restor Dent Endod. 2012;37(4):240-4. doi: 10.5395/rde.2012.37.4.240. [PubMed: 23430343].

6. Choudhary A, Garg R, Bhalla A, Khatri RK. Tooth fragment reattachment: An esthetic, biological restoration. J Nat Sci Biol Med. 2015;6(1):205-7. doi: 10.4103/0976-9668.149123. [PubMed: 25810662].

7. Jagannath-Torvi S, Kala M. Restore the natural - A review and case series report on reattachment. J Clin Exp Dent. 2014;6(5):e595-8. doi: 10.4317/jced.50948. [PubMed: 25674333].

8. Vaz VT, Presoto CD, Jordao KC, Paleari AG, Dantas AA, Segalla JC, et al. Fragment reattachment after atypical crown fracture in maxillary central incisor. Case Rep Dent. 2014;2014:231603. doi: 10.1155/2014/231603. [PubMed: 25610663].

9. Taguchi CM, Bernardon JK, Zimmermann G, Baratieri LN. Tooth fragment reattachment: a case report. Oper Dent. 2015;40(3):227-34. doi: 10.2341/14-034-T. [PubMed: 25535781].

10. Stokes AN, Hood JA. Impact fracture characteristics of intact and crowned human central incisors. J Oral Rehabil. 1993;20(1):89-95 [PubMed: 8429427].

11. van Dijken JW, Sjostrom S, Wing K. The effect of different types of composite resin fillings on marginal gingiva. J Clin Periodontol. 1987;14(4):185-9. [PubMed: 3294911].

12. VamsiKrishna R, Madhusudhana K, Swaroopkumarreddy A, Lavanya A, Suneelkumar C, Kiranmayi G. Shear bond strength evaluation of adhesive and tooth preparation combinations used in reattachment of fractured teeth: an ex-vivo study. I Indian Soc Pedod Prev Dent 2015;33(1):40-3. doi: 10.4103/0970-4388.148975. [PubMed: 25572372].

13. Hegde SG, Tawani GS, Warhadpande MM. Use of quartz fiber post for reattachment of complex crown root fractures: A 4-year followup. J Conserv Dent. 2014;17(4):389-92. doi: 10.4103/0972-0707.136519. [PubMed: 25125857].

14. Baratieri LN, Monteiro Jr S, Caldeira de Andrada MA. Tooth fracture reattachment: case reports. Quintessence Int. 1990;21(4):261-70. [PubMed: 2243926].

15. Kulkarni VK, Bhusari CP, Sharma DS, Bhusari P, Bansal AV, Deshmukh J. Autogenous tooth fragment reattachment: a multidisciplinary management for complicated crown-root fracture with biologic width violation. J Indian Soc Pedod Prev Dent. 2014;32(2):190-4. doi: 10.4103/0970-4388.131007. [PubMed: 24739925].

16. Patni P, Jain D, Goel G. A holistic approach to management of fractured teeth fragments: a case report. Oral Surg Oral Med Oral Pathol Oral Radiol Endod. 2010;109(5):e70-4. doi: 10.1016/j.tripleo.2009.12.045. [PubMed: 20416523]. 